\title{
Ear Recognition Based on 3D Keypoint Matching
}

\author{
Hui Zeng, Ji-Yuan Dong, Zhi-Chun Mu, Yin Guo \\ School of Information Engineering, University of Science and Technology Beijing, Beijing, China \\ hzeng@ustb.edu.cn
}

\begin{abstract}
This paper proposes a novel ear recognition approach based on 3D keypoint matching. At first, the 3D keypoints are detected using the shape index image and the scale space theory. Then two principal orientations are assigned and the normalized local range image is obtained, which can provide invariance to 3D rotation and transformation for the following local descriptor construction. Finally, we construct the 3D CSLBP features and use a coarse to fine strategy for 3D keypoint matching. The number of the matching points and their average EMD distances are used for 3D ear recognition. The proposed approach can reduce the amount of 3D data by 3D keypoint detection and local feature construction, and it doesn't need any expensive preprocessing steps. Compared with existing 2D or 3D LBP operators, the proposed 3D CS-LBP operator can not only remain the 3D LBP's powerful ability to describe the 3D structure information, but also reduce the histogram size and enhance its robustness to noise. Extensive experiments have performed to valid the efficiency of the proposed approach.
\end{abstract}

Keywords-3D ear recogntion; shape index; $3 D$ keypoint detection; $3 D$ center-symmetric $L B P$

\section{INTRODUCTION}

Ear recognition has attracted much attention in recent years. Compared with other popular human features, ear has many advantages, such as it has a rich and stable structure that changes little with age and does not suffer from changes in facial expression and so on. Especially, when we can't capture the well-posed face images, ear recognition technique becomes more important. Much work has been done in this field and those approaches roughly can be classified into two categories: 2D-based approaches and 3D-based approaches [1-5]. Compared with the 2D-based approaches, the 3D-based approaches are relatively insensitive to pose and lighting variations. So in recent years, more and more researchers began to pay more and more attention to the recognition approaches based on 3D ear data.

Up to now, only a few works about 3D ear recognition have been published. Pin Yan and Kevin W. Bowyer proposed an automatic 3D ear recognition system [3]. They use the iterative closest point (ICP) based 3D shape matching algorithm for ear recognition. Hui Chen and Bir Bhanu proposed a $3 \mathrm{D}$ ear recognition method based on local surface patch and ICP method [4-5]. Most of the existing 3D ear recognition methods adopt ICP algorithm for aligning the ear surfaces. For ICP algorithm based ear recognition method, a good initial estimation of the transformation is necessary to guarantee the convergence of the algorithm.

Although the discriminative feature based $3 \mathrm{D}$ ear recognition method has not been researched extensively, a large amount of work about discriminative feature based 3D face recognition methods have been published $[6,7]$. Huang et al proposed a 3D face recognition approach based on 3D local binary Patterns (3D LBP) operator. Although promising results have testified its effectiveness, the proposed approach need expensive preprocessing techniques such as registration and range image normalization. Mayo et al proposed a 3D face recognition approach based on point cloud rotations, multiple projections, and voted keypoint matching [9]. This approach didn't need any expensive processing techniques such as facial feature detection and 3D face registration, but the interval of the rotation angles can influence the final recognition results.

Inspired by the SIFT keypoint matching algorithm [10], we propose a novel 3D keypoint matching algorithm for 3D ear recognition. Compared with other existing 3D ear recognition approaches, our proposed approach doesn't need any expensive preprocessing and 3D ear registration processing which are indispensable steps and the amount of the 3D data can be reduced by detecting $3 \mathrm{D}$ keypoints and constructing local features using the proposed 3D Center-Symmetric LBP (3D CS-LBP) operator.

\section{3D EAR KEYPOINT DETECTION AND MATCHING BASED ON 3D CENTER-SYMMETRIC LBP}

\section{A. 3D Ear Keypoint Detection}

In this paper, the raw 3D ear points are arranged in strips in $(x, y)$ space with $z$ coordinate of each point indicating its depth. At first, we compute the shape indexes of the 3D ear data. Shape index $(S I)$ is a quantitative measure of the shape of a surface at a point $p[11]$. It is defined as:

$$
S I(p)=\frac{1}{2}-\frac{1}{\pi} \tan ^{-1} \frac{\kappa_{1}(p)+\kappa_{2}(p)}{\kappa_{1}(p)-\kappa_{2}(p)}
$$

where $\kappa_{1}$ and $\kappa_{2}$ are the principal curvatures of the surface, with $\kappa_{1} \geq \kappa_{2}$. With this definition, the range of the shape index is $[0,1]$. As the principal curvatures are invariable to rotation and transformation, the shape indexes are rotation and transformation invariant. Figure 1(a) shows the 3D surface mesh of an ear range data. Figure 1(b) shows the corresponding shape index image and its gray intensity values are proportionate to the shape index values of the $3 \mathrm{D}$ ear points.

Then the SIFT (Scale Invariant Feature Transform) keypoint detection algorithm is used on the shape index image [10]. Figure 2 shows the results of keypoint detection on two shape index images for one people, and the keypoints are marked with green dots. We can see that some feature points

978-1-4244-5900-1/10/\$26.00 @2010 IEEE 
corresponding to the same physical position appear in both images.

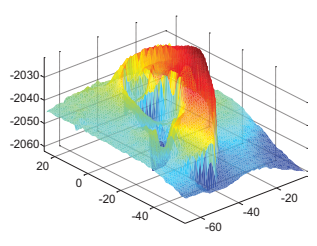

(a)

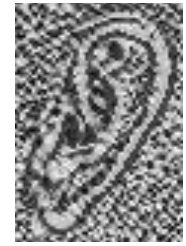

(b)
Figure 1. An example of an ear range data displayed as the 3D surface mesh (a) and its corresponding shape index image (b)

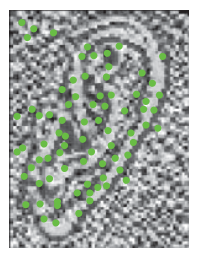

(a)

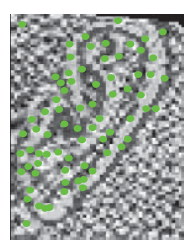

(b)
Figure 2. Two results of keypoint detection

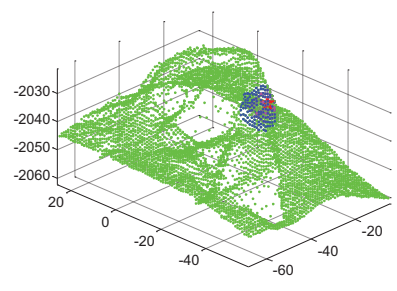

(a)

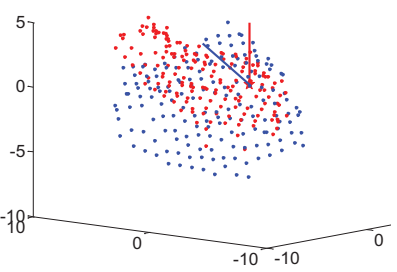

(b)
Figure 3. An example of the 3D keypoint and its neighborhood (a) and its key local regions and their surface normal before and after rotation (b)

\section{B. Principal Orientations Assignment For Each 3D Keypoint}

After 3D coordinates and scales of the keypoints have been obtained, we can determine the 3D key local region that consists of the keypoint and its neighbors. The neighborhood of the keypoint is determined according to its scale estimated in keypoint detection step. The neighbors of the 3D keypoint are the points that the distances between the keypoint and the points are less than $6 \times$ scale. As shown in Figure 3(a), a 3D keypoint is marked with a red pentagram and its neighbors are marked with blue dots.

First of all, we build the 3D local coordinate system of the keypoint. Let the location of the 3D keypoint be the origin of the local coordinate system, which can provide invariance to 3D translation for the local descriptor construction. Then we estimate two principal orientations to build unique 3D local coordinate system of the $3 \mathrm{D}$ key local region. The first principal orientation of the $3 \mathrm{D}$ keypoint is the normal of its local surface. Let the first principal orientation be the $z$ axis of the $3 \mathrm{D}$ local coordinate system. That is to say, we rotate the original coordinates of the $3 \mathrm{D}$ points in the key local region so that the normal of the transformed local surface is the $z$ axis of the 3D local coordinate. Figure 3(b) shows the point clouds before and after rotation and the red pentagram is the $3 \mathrm{D}$ keypoint that has been marked in Figure 3(a). The blue dots are the points of the key local region and the blue line is its surface normal before rotation. The red dots are the points of the rotated key local region and the red line is its surface normal.

Then we convert the 3D points of the transformed key local region into a local range image and their corresponding gray values are proportional to their $z$ coordinate values. Figure 5(a) shows the corresponding local range image of the rotated point cloud showed in Figure 4. The second principal orientation can be estimated using similar orientation assignment method for SIFT keypoints. For the converted local range image, a gradient orientation histogram is computed and the peak of the histogram is detected as the assigned second principal orientation. Let the second principal orientation be the $y$ axis of the $3 \mathrm{D}$ local coordinate system. Then we rotate the local range image so that the $v$ axis of the image coordinate system is parallel to the $y$ axis of the computed 3D local coordinate system. Figure 4(b) shows the rotated local range image corresponding to the range image showed in Figure 4(a). After the above steps, the $3 \mathrm{D}$ local coordinate of the $3 \mathrm{D}$ key local region can be determined uniquely and the normalized local range image can be obtained. All future operations are performed on the normalized local range images that have been transformed relative to their 3D local coordinate systems, which can provide invariance to $3 \mathrm{D}$ rotation and translation for the local descriptor construction.

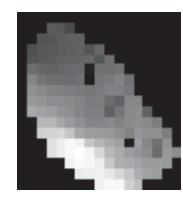

(a)

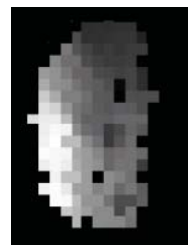

(b)
Figure 4. The local range image (a) and its corresponding normalized local range image $(b)$

\section{C. $3 D$ Center-Symmetric Local Binary Patterns (3D CS-LBP)}

The original LBP operator is introduced by Ojala et al. and has been proved a powerful means of texture analysis in 2D images [12]. The operator labels the pixels of an image by thresholding a $3 \times 3$ neighborhood of each pixel. Then the binary results are arranged clockwise and they can be transformed to decimal number. Finally the 256-bin histograms of the LBP labels can be constructed over the image as the texture descriptor. To reduce the histogram size of the LBP, Various extensions to the original LBP operator have been proposed, such as uniform LBP [12], Center-Symmetric LBP (CS-LBP) [13], and so on.

In recent years, with the development of $3 \mathrm{D}$ recognition technique, 3D LBP operator designed for the range image has been proposed. Experimental results have testified that it has greater power to encode the $3 \mathrm{D}$ structure information than $2 \mathrm{D}$ LBP. But the histogram size of 3D LBP is three times larger than that of 2D LBP. To address this problem, in this paper, we propose a new 3D Center-Symmetric LBP (3D CS-LBP) operator. At first, we review the 2D CS-LBP operator. The descriptor describes the result over the neighborhood as a binary number: 


$$
\begin{aligned}
& C S-L B P(x, y)=\sum_{i=0}^{(N / 2)-1} s\left(n_{i}-n_{i+(N / 2)}\right) 2^{i}, \\
& s(x)= \begin{cases}1 & x>T \\
0 & \text { otherwise }\end{cases}
\end{aligned}
$$

where $n_{i}(i=1, \cdots, N)$ correspond to the gray value of $N$ equally spaces pixels on a circle of radius $R$. Figure 5 illustrates the 2D CS-LBP feature construction method for a neighborhood of eight pixels.

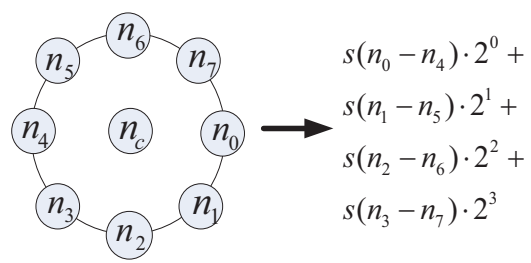

Figure 5. An example of 2D CS-LBP

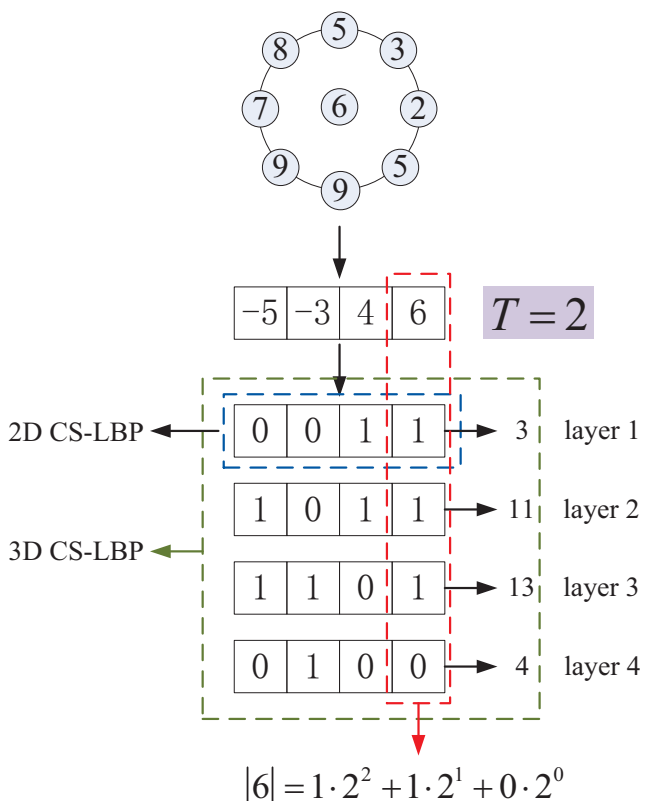

Figure 6. An example of 3D CS-LBP

Then we extend the 2D CS-LBP operator to 3D. As illustrated in Figure 6, the 3D CS-LBP operator has four layers and the first one is the same as the 2D CS-LBP. The last three layers are obtained by encoding the exact values of differences into binary units. Then four LBP Maps can be computed and four corresponding feature vectors $F_{i}(i=1,2,3,4)$ are formed from their histogram entries. For eight neighbors, 3D LBP proposed in [8] produces $4 \times 256\left(2^{8}\right)$ different binary patterns, whereas for 3D CS-LBP this number is only $4 \times 16\left(2^{4}\right)$. We can see that 3D CS-LBP operator can reduce the histogram size effectively. As the 3D CS-LBP operator uses the threshold to compute the feature, it is more robust to noise than 3D LBP operator. Finally each feature vector is normalized to unit length.

\section{3D Keypoint Matching}

In this paper, we use a coarse to fine strategy for 3D keypoint matching. Firstly, the corresponding shape index values of the $3 \mathrm{D}$ keypoints are used for coarse similarity measurement. Let $X_{i}$ and $Y_{j}$ be two 3D keypoints of different $3 \mathrm{D}$ ear models, and their corresponding shape index values are $S I_{i}$ and $S I_{j}$. Thus the distance between the two shape index values can be computed as follows:

$$
D_{i j}=\left|S I_{i}-S I_{j}\right|
$$

Then we determine the initial candidate matching keypoints according to the distance $D_{i j}$. If $D_{i j}$ is above the threshold, we can conclude that the two keypoints are not matching points. Otherwise, we will further compare their corresponding 3D LBP feature vectors to determine the final matching keypoints. Here we chose the Earth Mover's Distance (EMD) as the dissimilarity measurement of the 3D LBP feature vectors. The EMD is defined as the minimal cost that must be paid to transform one histogram into the other [14-15]. It has good performance to compute the distance between two histograms. The EMD distance between two 3D CS-LBP features can be computed as follows:

$$
D F_{i j}=\sum_{k=1}^{4} E M D\left(F_{i}^{k}, F_{j}^{k}\right)
$$

where $F_{i}^{k}$ and $F_{j}^{k}$ are the feature vectors corresponding to the 3D keypoint $X_{i}$ and $Y_{j}$, and $\operatorname{EMD}(a, b)$ denotes the EMD distance between the vector $a$ and $b$. The final matching points can be selected from the initial candidate matching keypoints according to the EMD distances of their 3D CS-LBP features.

\section{3D EAR RECOGNITION}

In this paper, the $3 \mathrm{D}$ ear recognition step is performed as follows. At first, we detect the 3D keypoints and construct the 3D CS-LBP feature vector of each keypoint for the gallery scans and the probe scan. Then we search the $3 \mathrm{D}$ matching keypoint between the probe scan and each gallery scan. Finally the nearest neighbor method is used for $3 \mathrm{D}$ ear recognition. Both the number of the final 3D matching points and their average EMD distances. The distance function between 3D ear model $E_{i}$ and $E_{j}$ is defined as:

$$
d\left(E_{i}, E_{j}\right)=\frac{\omega}{n u m+1}+(1-\omega) \frac{\sum_{k=1}^{n u m} D F_{i j}}{n u m}
$$

where num is the number of the final 3D matching points using the 3D CS-LBP descriptor, and $\omega$ is the weighting factor. The reason we add 1 to num in Equation (5) is if the number of the matching points is 0 , the denominator isn't 0 .

\section{EXPERIMENTAL RESULTS}

Our experiments are carried out on UND (University of Notre Dame) 3D ear database. To our best knowledge, this 
database is the largest 3D ear database till now. In this paper, we use a subset of UND 3D ear database, which consists of 830 scans from 415 subjects acquired with a Minolta Vivid 910 range scanner. For each scan, both one $640 \times 480$ 3D scan and one $640 \times 480$ color image were obtained at the same time. The distance from the subject sat to the sensor is about $1.5 \mathrm{~m}$. As the ear region detection isn't our research emphasis, we select the ear region by hand. In fact, we also can adopt the automatic ear detection methods proposed in [3] and [4]. For each subject, one scan is randomly selected for training; the residual one is used for testing. In the following experiments, the number of the neighbors is 8 , the threshold of the $2 \mathrm{D} \mathrm{CS}$ LBP and 3D CS-LBP is 2, and the weighting factor $\omega$ is 0.7 . These parameters are selected by analyzing a great deal of experimental results.

In order to investigate the effectiveness of the proposed 3D CS-LBP operator, we compare it with other operators. Table 1 gives the recognition rates of different approaches. The 2D LBP operator, 2D CS-LBP operator, 3D LBP operator and 3D CS-LBP operator are used to describe the 3D key local regions respectively. From Table 1 we can see that, "3D CS-LBP" approach has the best recognition results and the performance of the 3D feature based approaches are better than that of the two 2D feature based approaches. That is because the 3D LBP feature has great power to encode the 3D structure information than the 2D LBP feature. As the CS-LBP operator is more robust to noise than LBP operator, the recognition rate of " $2 \mathrm{D}$ CS-LBP" approach is higher than that of "2D LBP" approach and the recognition rate of "3D CS-LBP" approach is higher than that of "3D LBP" approach.

TABLE I. RECOGNITION RATES OF DIFFERENT APPROACHES

\begin{tabular}{|c|c|c|c|c|c|}
\hline \multirow{2}{*}{ Approach } & \multicolumn{5}{|c|}{ Recognition Rate } \\
\cline { 2 - 6 } & Rank1 & Rank2 & Rank3 & Rank4 & Rank5 \\
\hline 2D LBP & $86.02 \%$ & $86.51 \%$ & $86.51 \%$ & $86.75 \%$ & $87.47 \%$ \\
\hline 2D CS-LBP & $87.95 \%$ & $88.19 \%$ & $88.43 \%$ & $88.92 \%$ & $89.40 \%$ \\
\hline 3D LBP & $94.70 \%$ & $95.18 \%$ & $95.42 \%$ & $95.66 \%$ & $96.14 \%$ \\
\hline 3D CS-LBP & $96.39 \%$ & $96.63 \%$ & $96.63 \%$ & $97.11 \%$ & $97.59 \%$ \\
\hline
\end{tabular}

\section{CONCLUSIONS}

This paper present a novel ear recognition approach based on 3D keypoint matching. The main contribution of this paper includes two aspects: 1) Compared with other existing 3D ear recognition approaches, our proposed approach can reduce the amount of 3D data by detecting 3D keypoints and constructing feature vectors using 3D CS-LBP operator. It does not rely on any expensive preprocessing techniques such as physiological detection (ear pit, crus of helix etc) and 3D ear registration. Moreover, our proposed recognition approach is robust to pose variations and partial occlusions. 2) Compared with existing 2D LBP operators, the proposed 3D CS-LBP operator has greater power to describe the 3D structure information. Compared with the 3D LBP operator proposed in [8], the 3D CS-LBP operator can reduce the histogram size and enhance its robustness to noise. Our experimental results have shown that the 3D CS-LBP operator has greater power to encode the $3 \mathrm{D}$ structure information than other existing $2 \mathrm{D}$ or $3 \mathrm{D}$ LBP operators.

\section{ACKNOWLEDGMENT}

This paper is supported by the Open Program of the National Laboratory of Pattern Recognition (NLPR) (No.0810), the National Natural Science Foundation of China under the Grant No. 60973064 and the Beijing Natural Science Foundation under the Grant No. 4102039.

\section{REFERENCES}

[1] D. J. Hurley, B. Zrbab-Zavar and M. S. Nixon, The Ear as a Biometric, Handbook Of Biometrics, Springer, 2008, pp. 131-150.

[2] Kyong Chang and Kevin W. Bowyer, Sudeep Sarkar and Barnabas Victor, Comparison and Combination of Ear and Face Images in Appearance-Based Biometrics, IEEE Transactions on Pattern Analysis And Machine Intelligence, September 2003, 25(9), pp. 1160-1165.

[3] Ping Yan and Kevin W. Bowyer. Biometric Recognition Using 3D Ear Shape, IEEE Transactions on Pattern Analysis And Machine Intelligence, August 2007, 29(8), pp. 1297-1308.

[4] Hui Chen and Bir Bhanu, Human Ear Recognition in 3D. IEEE Transactions on Pattern Analysis And Machine Intelligence, April 2007, 29(4), pp. 718-737.

[5] Hui Chen and Bir Bhanu. Efficient Recognition of Highly Similar 3D Objects in Range Images, IEEE Transactions on Pattern Analysis And Machine Intelligence, January 2009, 31(1), pp. 172-179.

[6] Andrea F. Abate, Michele Nappi, Daniel Riccio and Gabriele Sabatino, 2D and 3D face recognition: A survey, Pattern Recogntion, October 2007, 28(14), pp. 1885-1906.

[7] Kyong I. Chang, Kevin W. Bowyer and Patrick J. Flynn, An Evaluation of Multimodal 2D+3D Face Biometrics, IEEE Transactions on Pattern Analysis And Machine Intelligence, April 2005, 27(4), pp. 619-624.

[8] Yonggang Huang, Yunhong Wang and Tieniu Tan, combining Statistics of Geometrical and Correlative Features for 3D Face Recognition, the $17^{\text {th }}$ British Machine Vision Conference, 2006, pp. 879-888.

[9] Michael Mayo, Edmond Zhang. 3D Face Recognition Using Multiview Keypoint Matching. Proceedings of the IEEE International Conference on Advanced Video and Signal Surveillance, 2009, pp. 290-295.

[10] D. Lowe., Distinctive Image Features from Scale-Invariant Keypoints, International Journal of Computer Vision, 2004, 60(2), pp.91-110.

[11] C. Dorai and A. Jain, COSMOS - A Representation Scheme for 3D Free-Form Objects, IEEE Transactions on Pattern Analysis And Machine Intelligence, October 1997, 19(10), pp. 1115-1130

[12] Ojala T, Pietikäinen M and Mäenpää T, Multiresolution gray-scale and rotation invariant texture classification with Local Binary Patterns. IEEE Transactions on Pattern Analysis and Machine Intelligence, 2002, 24(7), pp. 971-987.

[13] M. Heikkilä, M. Pietikäinen, C. Schmid, Description of interest regions with local binary patterns, Pattern Recognition, 2009, 42(3), pp. 425436.

[14] Rubner Y., Tomasi C. and Guibas L.J.. The earth mover's distance as a metric for image retrieval. International Journal of Computer Vision. 2000, 40(2), pp.99-121.

[15] Ling H. and Okada K.. An Efficient Earth Mover's Distance Algorithm for Robust Histogram Comparison. IEEE Transactions on Pattern Analysis and Machine Intelligence. 2007, 29(5), pp.840-853. 